\title{
Successful management of an inadvertent excessive treprostinil overdose
}

This article was published in the following Dove Press journal:

Drug Design, Development and Therapy

20 March 2013

Number of times this article has been viewed

\author{
Wolfgang Hohenforst- \\ Schmidt ${ }^{\prime}$ \\ Juergen Hornig ${ }^{2}$ \\ Norbert Friedel ${ }^{3}$ \\ Paul Zarogoulidis ${ }^{1,4}$ \\ Konstantinos Zarogoulidis ${ }^{4}$ \\ Johannes Brachmann' \\ 'Il Medical Clinic, Coburg Clinic, \\ University of Würzburg, Coburg, \\ Germany; ${ }^{2}$ Department of Cardiology, \\ Bayreuth Clinic, University of \\ Erlangen, Bayreuth, Germany; \\ ${ }^{3}$ Department of Cardiac Surgery, \\ "Bayreuth Clinic", University of \\ Erlangen, Bayreuth, Germany; \\ ${ }^{4}$ Pulmonary Department, \\ G Papanikolaou, General Hospital, \\ Aristotle University of Thessaloniki, \\ Thessaloniki, Greece
}

\begin{abstract}
Pulmonary hypertension is defined by $25 \mathrm{mmHg}$ pressure at rest, and $35 \mathrm{mmHg}$ pressure at exercise, in the pulmonary arteries. Hypertension either primary or secondary. The exact prevalence of all types of pulmonary hypertension is not yet known. We present a case of a 58-year-old female patient suffering from CREST syndrome, Raynaud's syndrome, esophageal motility impairment, and severe pulmonary hypertension who had previously obtained a specially developed implantable pump, named Lenus Pro $^{\circledR}$, to facilitate continuous parenteral treatment of pulmonary arterial hypertension with treprostinil. Treprostinil is a prostanoid derivative with very stable physiochemical properties which allows subcutaneous treatment of pulmonary arterial hypertension in the outpatient. Treprostinil is normally dosed individually in a range of 0.6 to $50 \mathrm{ng} / \mathrm{kg} /$ minute. In the underlying case, a dose of more than $100 \mathrm{mg}$ given over 1 minute is equivalent to a 1000 fold overdose. The patient's critical condition required installment of a central venous access, full monitoring, sedation, oxygen nasal tube, fluid balance, and parenteral nutrition. The patient could be hemodynamically stabilized within 24 hours after the overdose. After 6 days of recovery, the patient left the hospital with no remaining health impairment.
\end{abstract}

Keywords: pulmonary hypertension, ICU, overdose, treprostinil, Raynaud's syndrome, CREST syndrome

A 58-year-old female patient suffering from CREST syndrome, Raynaud's syndrome, esophageal motility impairment, and severe pulmonary hypertension had previously obtained a specially developed implantable pump, named Lenus Pro ${ }^{\circledR}$, to facilitate continuous parenteral treatment of pulmonary arterial hypertension with treprostinil.

Implantable gas propelled pumps have been used for over two decades for parenteral administration of analgesic and chemotherapeutic agents and are highly accurate and technically reliable. ${ }^{1-4}$ The Lenus Pro ${ }^{\circledR}$ (Tricumed Medizintechnik GmbH, Germany, Frittlingen, Germany) requires a monthly refill performed under sterile conditions according to a standardized procedure. Lack of adherence to this standardized refill procedure resulted in the instantaneous administration of a one monthly treprostinil supply into the subcutaneous tissue instead of into the pump reservoir. Treprostinil is normally dosed individually in the range of 0.6 to $50 \mathrm{ng} / \mathrm{kg} /$ minute. In the underlying case, a dose of more than $100 \mathrm{mg}$ given over 1 minute is equivalent to a 1000 fold overdose. Within minutes the patient suffered from circulatory collapse which was antagonized by adrenaline and terlipressin, and there was an immediate referral to a cardiosurgically focused intensive care unit (ICU) (blood pressure, 71/48 mmHg; saturation $\mathrm{O}_{2}, 68 \%$; partial arterial $\mathrm{O}_{2}, 35 \mathrm{mmHg}$; partial arterial $\mathrm{CO}_{2}, 24 \mathrm{mmHg}, \mathrm{S}$-Creatine, $1.5 \mathrm{mg} / \mathrm{dL}$; respiratory frequency, 33/min; mean heart frequency, 137/min with a variance between 100/min and 160/min). submit your manuscript $\mid$ www.dovepress.com

Dovepress

http://dx.doi.org/10.2147/DDDT.S4277|
Drug Design, Development and Therapy 2013:7 161-165 
Upon ICU admission, treprostinil can be partially removed by puncture of the injection site and subsequent repeated rinsing of the area. Excessive hypotension was fought with very high doses of vasopressor agents. Cardiac arrhythmias (supraventricular arrhythmias [in the majority] as well as ventricular arrhythmias [different ventricular extrasystoles (VES) of different origin, short ventricular tachycardias (VTs), no sustained VTs, or R-on-Ts waves]) were managed by amiodarone, calcium, and potassium substitution. The patient could be hemodynamically stabilized within 24 hours after the overdose. Thereafter, specific pulmonary arterial hypertension (PAH) therapy had to be gradually reinitiated and concomitantly vasopressors were phased out. After 6 days of recovery the patient left the hospital with no remaining health impairment.

Treprostinil is a prostanoid derivative with very stable physiochemical properties which allows subcutaneous treatment of pulmonary arterial hypertension in the outpatient. ${ }^{5}$ Treprostinil subcutaneous (sc) administration is devoid of line sepsis risk but does frequently come with site reactions which may be very painful. ${ }^{6}$ Moreover, bearing of an external pump for many patients does pose a stigmatic burden. For this reason parenteral treprostinil, despite being a highly effective treatment, is frequently started at a very late stage of the disease, at a time when oral combination regimen loses efficacy. To encourage a timely introduction of parenteral prostanoid therapy, the implantable Lenus Pro $^{\circledR}$ pump was developed.

The closed system feature and the central venous drug administration avoid both line infections and infusion site reactions. The system's advantages are at the expense of a professional service staff requirement to aid implantation and refill procedures. Refill procedures need to be done once monthly in accordance with a standardized operation procedure and sterile conditions which require specially trained health care professionals.

Accidents are usually a chain of unfortunate events. Analysis therefore allows avoidance of similar occurrences in the future. In the underlying case, the patient's pump refill procedures had been delegated to a resident medical specialist in the geographical region. Both the medical specialist and the local experienced nurse assistant had obtained the obligatory pump refill training by the pump company and had already successfully conducted 15 refill procedures. The experienced nurse assistant was used to doing the manual refill procedure while the medical specialist followed the process for plausibility control. The technical features of the pump provide good feedback for successful access to the pump reservoir. Upon penetration of the reservoir septum, the residual volume is returned into the accessing syringe at a pressure of 2 bar. This high pressure reflux clearly confirms the correct position of the refill needle and allows the readily reversible instillation of a monthly $40 \mathrm{~mL}$ drug solution charge which needs to be injected against an elastic resistance of 2 bar.

In the reported case, the entire monthly treprostinil dose was injected subcutaneously, ie, "para" pump. Within minutes the patient started to suffer from typical prostanoid side effects, and rapid and severe blood pressure decay was accompanied by tachycardia. The patient was immediately administered a vasopressor and transferred to a nearby cardiology ICU. Hospitalization occurred within 1 hour of the accidental drug administration. Upon admission, efforts to recover the drug by means of local puncture, aspiration, repeated topical instillation, and recovery with $0.9 \% \mathrm{NaCl}$ were made. Accordingly, a nonquantifiable amount of treprostinil was recovered.

The patient's critical condition required installment of a central venous access, full monitoring, sedation, oxygen nasal tube, fluid balance, and parenteral nutrition.

Symptoms such as severe hypotension, tachycardia, ventricular and supraventricular arrhythmias, motor unrest, dyspnea, and flush dominated the following 12 hours. Since treprostinil may not be reliably eliminated by hemodialysis (plasma levels may be reduced by $25 \%$ during hemodialysis, but without impact on efficacy) and there is no specific antidote available, management of the situation was done symptomatically. ${ }^{7,8}$ Severe hypotension was initially controlled with $500 \mathrm{~mL}$ of $6 \%$ hydroxyethyl starch. Concomitant infusion of high dose norepinephrine (up to $20 \mathrm{mg}$ /hour) and brief infusions of terlipressin (at $0.5 \mathrm{mg}$ /hour) was initiated.

Hemodynamic stabilization over the time period of 60 hours following admission is shown in Figures 1 and 2. Severe arrhythmias within the first 8 hours of admission were managed by amiodarone $(150 \mathrm{mg} / 100 \mathrm{~mL}$, brief infusion) followed by $\mathrm{KCl}$ (up to $10 \mathrm{mval} /$ hour) and regular insulin (5-10 IU/hour) via a perfusor. Fluid, electrolyte therapy, and acid-base balance was maintained according to respective requirements.

Motor unrest, agitation, and pain within 6 hours of admission were managed with intermittent midazolam, propofol and piritramide. Pantoprazole (40 mg) and acetylcysteine (300 mg) were administered intravenously once and twice per day, respectively, over the entire intensive care period. Nutrition for the first day following admission was provided by Sterofundin G-Infusionslösung ${ }^{\circledR}$ (B Braun, Melsungen, Germany). The most critical time period following the overdose proved to be the first 8 hours. Renal function, determined 


\section{Systemic hemodynamic changes}

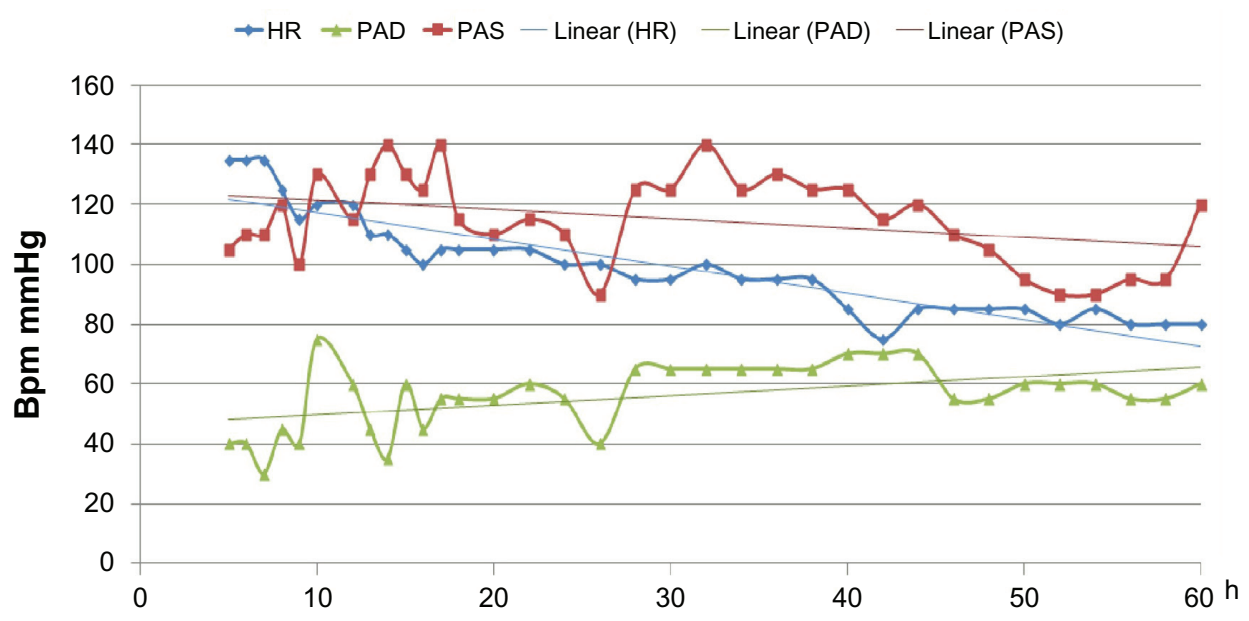

Specific PAH therapy reintroduction
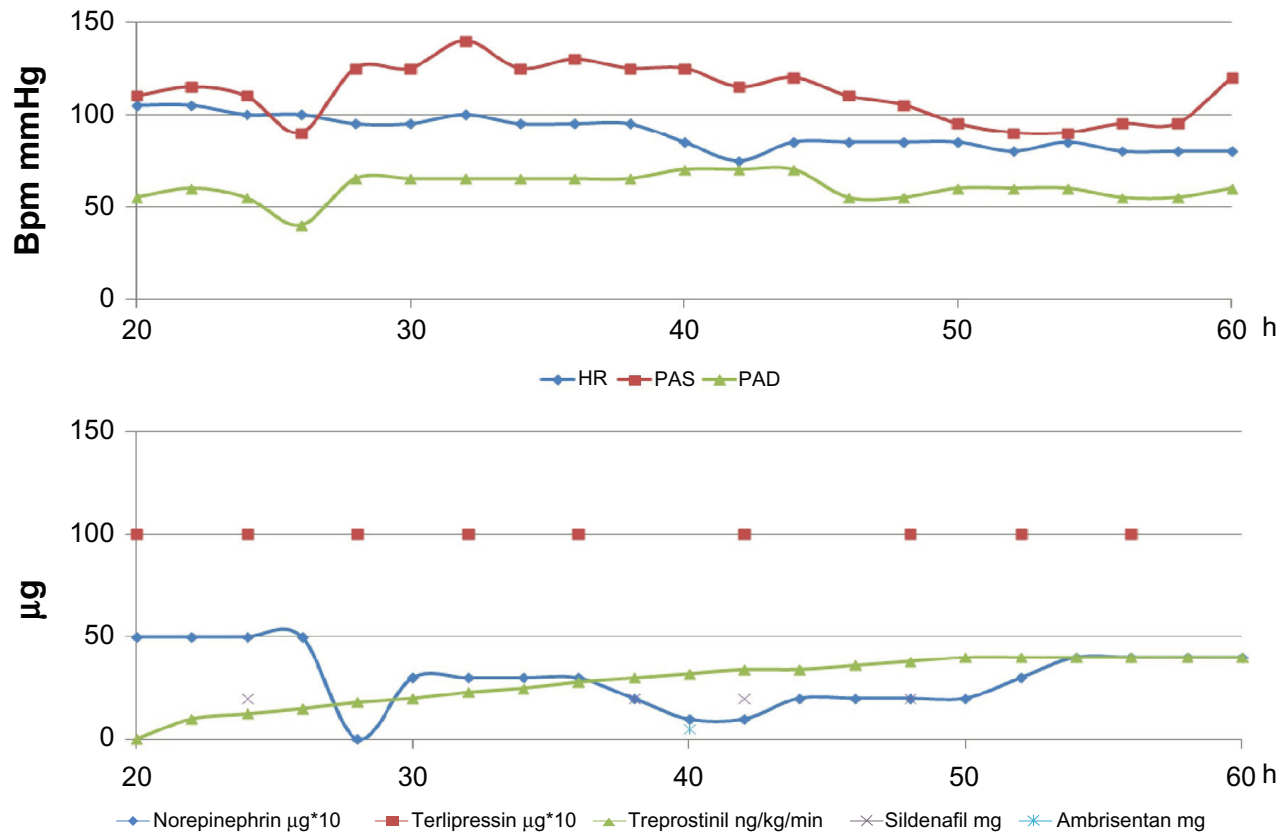

Figure I Pharmaceuticals administered correlated with Bpm (x-axis) and time (y-axis).

Abbreviation: Bpm, blood pressure per minute; HR, heart rate; PAS, pulmonary arterial systolic pressure; PAD, pulmonary arterial diastolic pressure; PAH, pulmonary arterial hypertension.

by urine recovery via a catheter, rendered volumes of 30,50 , $100,20,300,200,450$, and $300 \mathrm{~mL}$ per hour.

Eighteen hours after admission, at a time when the patient's peripheral blood pressure had been stabilized, central venous pressure monitoring rendered increasing values of up to $18 \mathrm{mmHg}$. At this point, specific oral and parenteral PAH treatment was reinitiated. Treprostinil infusion started at $10 \mathrm{ng} / \mathrm{kg} /$ minute and was increased hourly by 2-3 ng $/ \mathrm{kg} /$ minute until the target dosage of $40 \mathrm{ng} / \mathrm{kg} /$ minute had been reached. The balance of specific PAH therapy versus administration of vasoconstrictors is shown in Figure 1.
After 3 days, terlipressin was stopped and prostanoid therapy was again administered via the implantable pump. Norepinephrine was intermittently given and then phased out on day 5. The patient left the hospital on day 6. By then all overdose related symptoms had abated and sustained damage could be ruled out.

Treprostinil has been used for the treatment of PAH related symptoms for more than a decade. The drug is a prostanoid with excellent physiochemical stability which allows inhalation and parenteral routes of administration. Due to the fact that treprostinil is administered under a 


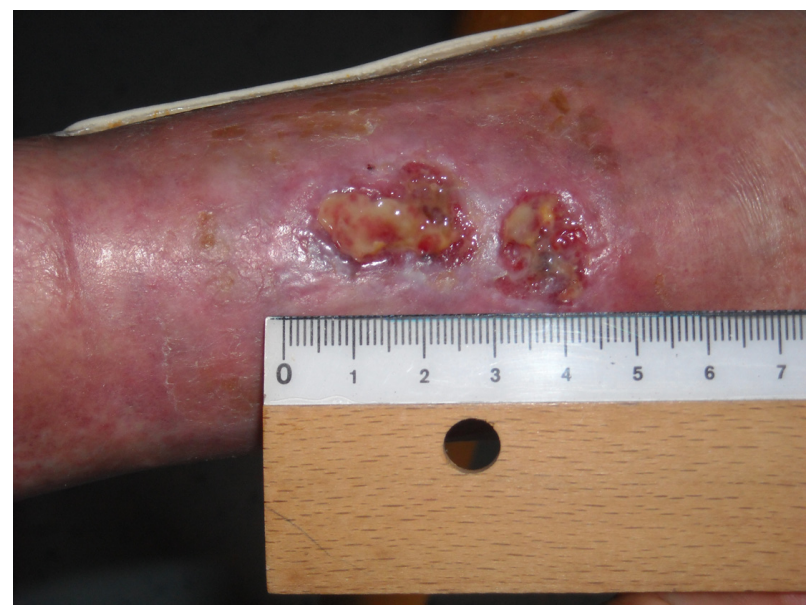

Figure 2 Lesions upon admission.

strongly guided procedure, to date there are very few overdose reports available. The current case, in terms of the actually administered dose, was entirely unprecedented. Hemodynamic stabilization following inadvertent excessive treprostinil overdose may be done with vasopressors such as norepinephrine and terlipressin. The respective dosages required are very high and even above the range used for hemodynamic management of sepsis. ${ }^{9,10}$ Treprostinil does

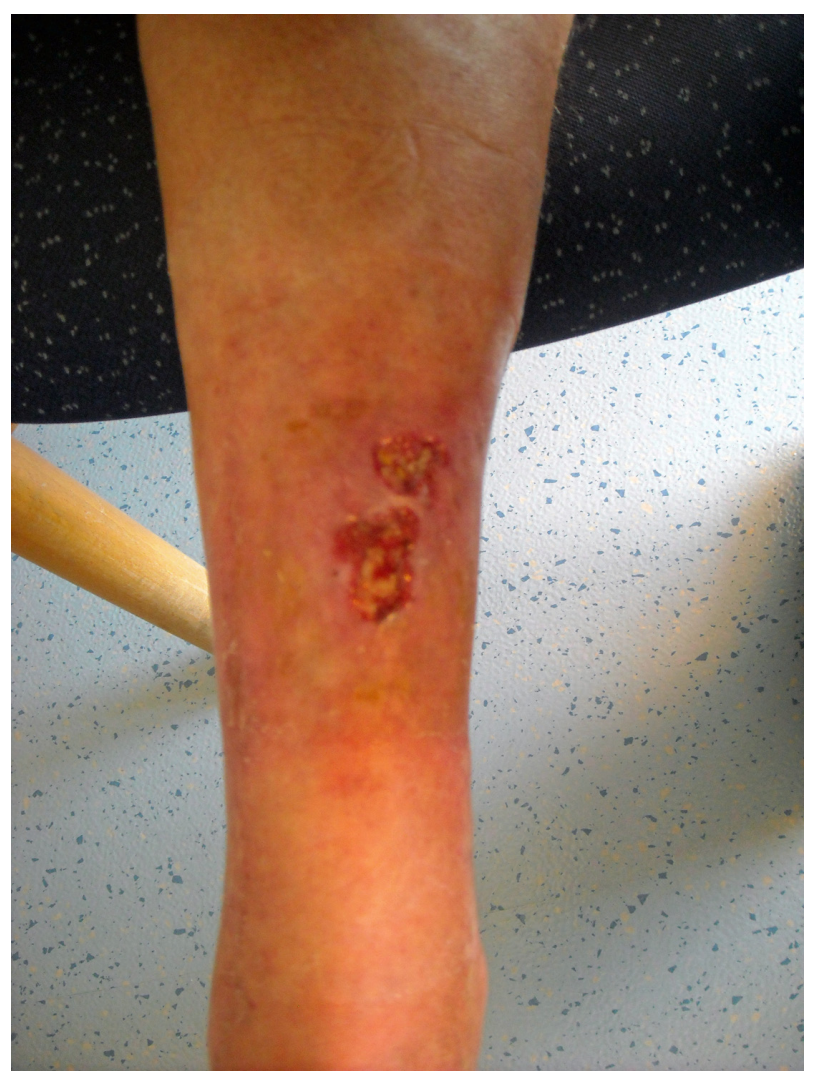

Figure 3 Leg lesion upon admission.

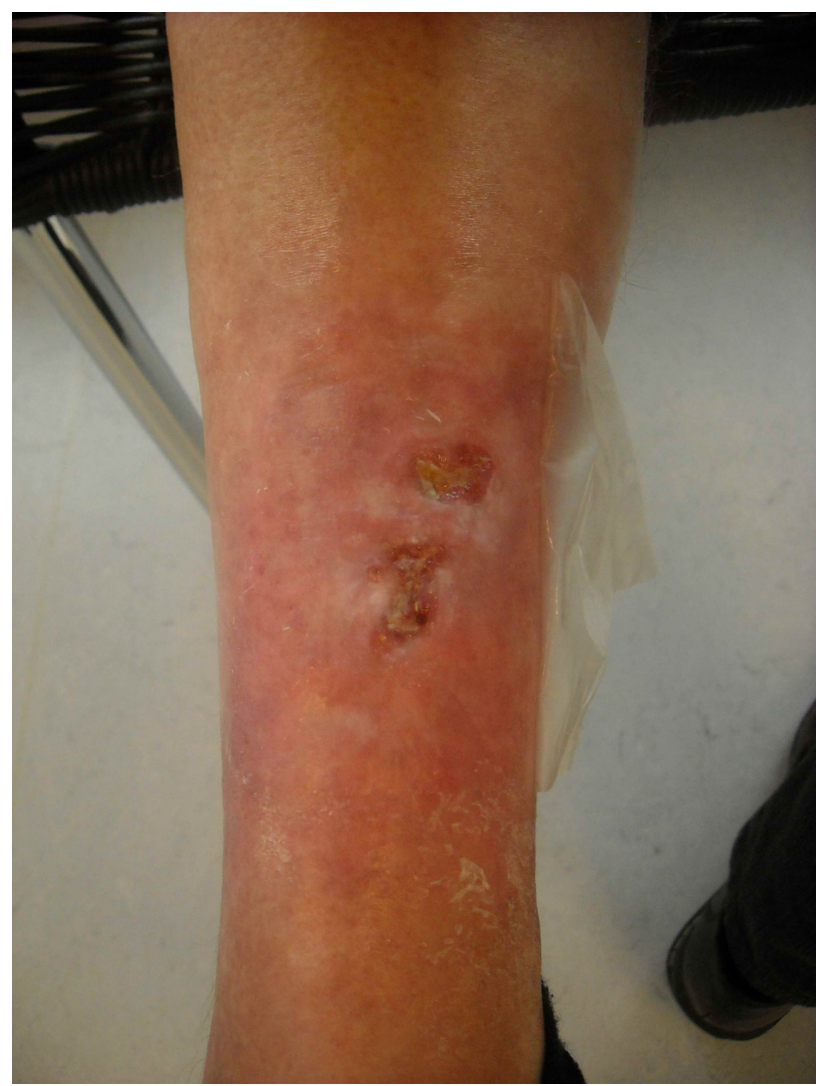

Figure 4 Leg lesion upon follow up.

have a half-life beyond 3 hours. Provided that the elimination mechanism is nonsaturable, drug elimination should have been completed after 12-16 hours. In the underlying case, the local depot/excessive drug amount in the body, however, did induce hemodynamic effects well beyond this time period. The most critical period was the first 8 hours following inadvertent administration of an overdose 1000 times above the therapeutic level. The circumstance that circulation could still be stabilized indicates that the maximum effect(s) of treprostinil seem to be limited by a ceiling effect due to exhaustion of receptor reserve or to receptor downregulation. Interestingly, after 18 hours the pulmonary circulation did require restart of $\mathrm{PAH}$ specific treatment while peripheral circulation still required vasopressor support. However, in this context, a distinction between low blood pressure due to lack of peripheral resistance and myocardial insufficiency is hard to make since norepinephrine influences the myocardium via the $\beta 1$ pathway and peripheral resistance vessels via $\alpha 1$ receptors. Based on the speed of the clinical improvement, the elimination of treprostinil does not seem to be saturable.

The filling procedure of the Lenus Pro ${ }^{\circledR}$ pump, despite being technically simple, should only be performed by well 
and regularly trained personnel. Treprostinil seems to have a very wide therapeutic margin with respect to the occurrence of fatal drug reactions. Hemodynamic effects of excessive treprostinil dosages may be antagonized by vasopressors such as norepinephrine or terlipressin.

\section{Conclusion}

The message for the clinical physician is summarized as follows: when you puncture the subcutaneous rubber on top of the drug bag incorporated in the pump, as the first step you have to aspirate the rest of the "old" filling volume (2-4 mL) so you can be sure - not only by feeling - that the needle is inside the rubber as the "door" to the bag. As a second step, during filling with the new drug using a syringe, you have to overcome a certain resistance due to the fact that the flexible bag is incorporated in spring blades of titanium blades which have to be pulled apart, building up the gas driven pressure to guarantee that this pump is working without electrical energy. This specific resistance is much higher than subcutaneous resistance, eg, when you empty a syringe subcutaneously. As a third step, before giving the whole "new" volume, it is necessary that you fill and aspirate a few milliliters. Following these rules, it is impossible to refill incorrectly. In cases of treprostinil intoxication, PAH specific therapy needs to be restarted and titrated up after 18-24 hours (Figures 3 and 4).

\section{Disclosure}

The authors declare no conflict of interest in this work.

\section{References}

1. Ebel H, Buschmann D, Conzen M, Oppel F. Initial experiences with implantable pump systems for intrathecal therapy of chronic pain conditions. Nervenarzt. 1993;64(7):468-473. German.

2. Ethans KD, Schryvers OI, Nance PW, Casey AR. Intrathecal drug therapy using the Codman Model 3000 Constant Flow Implantable Infusion Pumps: experience with 17 cases. Spinal Cord. 2005;43(4):214-218.

3. Desole S, Velik-Salchner C, Fraedrich G, Ewert R, Kahler CM Subcutaneous implantation of a new intravenous pump system for prostacyclin treatment in patients with pulmonary arterial hypertension. Heart Lung. 2012;41(6):599-605.

4. Porpodis K, Konoglou M, Zarogoulidis P, et al. Pulmonary thromboendarterectomy after treatment with treprostenil in a chronic thromboembolic pulmonary hypertension patient: a case report. Int J Gen Med. 2011;4:767-772

5. Phares KR, Weiser WE, Miller SP, Myers MA, Wade M. Stability and preservative effectiveness of treprostinil sodium after dilution in common intravenous diluents. Am J Health Syst Pharm. 2003;60(9):916-922.

6. Skoro-Sajer N, Lang I. Treprostinil for the treatment of pulmonary hypertension. Expert Opin Pharmacother. 2008;9(8):1415-1420.

7. Bailie GR, Mason NA. 2012 Dialysis of Drugs, 5th ed. Saline, Michigan, USA. Renal Pharmacy Consultants, LLC; 2012.

8. Yigla M. Impact of hemodialysis therapy on blood levels of treprostinil. in a PAH Patient with End-Stage Renal Disease Current Medical Literature. 2012;3(s2):17.

9. Martin C, Papazian L, Perrin G, Saux P, Gouin F. Norepinephrine or dopamine for the treatment of hyperdynamic septic shock? Chest. 1993; 103(6):1826-1831.

10. Svoboda P, Scheer P, Kantorova I, et al. Terlipressin in the treatment of late phase catecholamine-resistant septic shock. Hepatogastroenterology. 2012;59(116):1043-1047.

\section{Publish your work in this journal}

Drug Design, Development and Therapy is an international, peerreviewed open-access journal that spans the spectrum of drug design and development through to clinical applications. Clinical outcomes, patient safety, and programs for the development and effective, safe, and sustained use of medicines are a feature of the journal, which

\section{Dovepress}

has also been accepted for indexing on PubMed Central. The manuscript management system is completely online and includes a very quick and fair peer-review system, which is all easy to use. Visit http://www.dovepress.com/testimonials.php to read real quotes from published authors. 